\title{
Elementary Number Theory Problems. Part I
}

\author{
Adam Naumowicz (iD \\ Institute of Informatics \\ University of Białystok \\ Poland
}

Summary. In this paper we demonstrate the feasibility of formalizing recreational mathematics in Mizar (1], 2] ) drawing examples from W. Sierpinski's book "250 Problems in Elementary Number Theory" [4. The current work contains proofs of initial ten problems from the chapter devoted to the divisibility of numbers. Included are problems on several levels of difficulty.

MSC: 11A99 68V20 03B35

Keywords: number theory; recreational mathematics

MML identifier: NUMBER01, version: 8.1.09 5.60.1374

\section{Problem 1}

One can verify that there exists an integer which is positive.

Now we state the propositions:

(1) Let us consider a positive integer $n$. Then $n+1 \mid n^{2}+1$ if and only if $n=1$.

Proof: If $n+1 \mid n^{2}+1$, then $n=1$ by [6, (2)].

(2) Let us consider integers $i, n$. If $|i|=n$, then $i=n$ or $i=-n$.

(3) Let us consider a natural number $n$. Suppose $n \mid 24$. Then

(i) $n=1$, or

(ii) $n=2$, or

(iii) $n=3$, or 
(iv) $n=4$, or

(v) $n=6$, or

(vi) $n=8$, or

(vii) $n=12$, or

(viii) $n=24$.

(4) Let us consider an integer $t$. Suppose $t \mid 24$. Then

(i) $t=-1$, or

(ii) $t=1$, or

(iii) $t=-2$, or

(iv) $t=2$, or

(v) $t=-3$, or

(vi) $t=3$, or

(vii) $t=-4$, or

(viii) $t=4$, or

(ix) $t=-6$, or

(x) $t=6$, or

(xi) $t=-8$, or

(xii) $t=8$, or

(xiii) $t=-12$, or

(xiv) $t=12$, or

(xv) $t=-24$, or

(xvi) $t=24$.

The theorem is a consequence of (3) and (2).

\section{Problem 2}

Now we state the proposition:

(5) Let us consider an integer $x$. Suppose $x-3 \mid x^{3}-3$. Then

(i) $x=-21$, or

(ii) $x=-9$, or

(iii) $x=-5$, or

(iv) $x=-3$, or 

(v) $x=-1$, or
(vi) $x=0$, or
(vii) $x=1$, or
(viii) $x=2$, or
(ix) $x=4$, or
(x) $x=5$, or
(xi) $x=6$, or
(xii) $x=7$, or
(xiii) $x=9$, or
(xiv) $x=11$, or
(xv) $x=15$, or
(xvi) $x=27$.

The theorem is a consequence of (4).

\section{Problem 3}

Now we state the proposition:

(6) $\left\{n\right.$, where $n$ is a positive integer : $5 \mid 4 \cdot\left(n^{2}\right)+1$ and $\left.13 \mid 4 \cdot\left(n^{2}\right)+1\right\}$ is infinite.

Proof: Set $S=\left\{n\right.$, where $n$ is a positive integer : $5 \mid 4 \cdot\left(n^{2}\right)+1$ and $\left.13 \mid 4 \cdot\left(n^{2}\right)+1\right\}$. Define $\mathcal{F}$ (natural number) $=65 \cdot \$_{1}+56$. Consider $f$ being a many sorted set indexed by $\mathbb{N}$ such that for every element $n$ of $\mathbb{N}, f(n)=\mathcal{F}(n)$. Set $R=\operatorname{rng} f . R \subseteq S$. For every element $m$ of $\mathbb{N}$, there exists an element $n$ of $\mathbb{N}$ such that $n \geqslant m$ and $n \in R$.

\section{Problem 4}

Now we state the proposition:

(7) Let us consider a positive integer $n$. Then $169 \mid 3^{3 \cdot n+3}-26 \cdot n-27$.

PROOF: Reconsider $k=n$ as a natural number. Define $\mathcal{P}$ [natural number] $\equiv$ $169 \mid 3^{3 \cdot \$_{1}+3}-26 \cdot \$_{1}-27$. For every natural number $k$ such that $1 \leqslant k$ holds $\mathcal{P}[k]$. 


\section{Problem 5}

Now we state the proposition:

(8) Let us consider a natural number $k$. Then $19 \mid 2^{2^{6 \cdot k+2}}+3$.

\section{Problem 6 (due to Kraitchik)}

Now we state the proposition:

(9) $13 \mid 2^{70}+3^{70}$.

\section{Problem 7}

Now we state the propositions:

(10) $11 \cdot 31 \cdot 61 \mid 20^{15}-1$.

(11) Let us consider an integer $a$, and a natural number $m$. Then $a-1 \mid a^{m}-1$. Proof: Define $\mathcal{P}$ [natural number] $\equiv a-1 \mid a^{\$_{1}}-1$. For every natural number $k, \mathcal{P}[k]$.

(12) Let us consider a natural number $a$, a positive integer $m$, and a finite 0 -sequence $f$ of $\mathbb{Z}$. Suppose $a>1$ and len $f=m-1$ and for every natural number $i$ such that $i \in \operatorname{dom} f$ holds $f(i)=a^{i+1}-1$. Then $a^{m}-1 \operatorname{div}(a-$ 1) $=\sum f+m$.

ProOF: Define $\mathcal{P}$ [natural number] $\equiv$ for every finite 0 -sequence $f$ of $\mathbb{Z}$ such that len $f=\$_{1}$ and for every natural number $i$ such that $i \in \operatorname{dom} f$ holds $f(i)=a^{i+1}-1$ holds $a^{\$_{1}+1}-1 \operatorname{div}(a-1)=\sum f+\left(\$_{1}+1\right) \cdot \mathcal{P}[0]$. For every natural number $k, \mathcal{P}[k]$.

\section{Problem 8}

Now we state the proposition:

(13) Let us consider a positive integer $m$, and a natural number $a$. Suppose $a>1$. Then $\operatorname{gcd}\left(a^{m}-1 \operatorname{div}(a-1), a-1\right)=\operatorname{gcd}(a-1, m)$.

Proof: Reconsider $m_{0}=m$ as a natural number. Reconsider $m_{1}=m_{0}-1$ as a natural number. Define $\mathcal{F}$ (natural number) $=a^{\$_{1}+1}-1$. Consider $f$ being a finite 0 -sequence such that len $f=m_{1}$ and for every natural number $i$ such that $i \in m_{1}$ holds $f(i)=\mathcal{F}(i)$ from [5, Sch.2]. $\operatorname{rng} f \subseteq \mathbb{Z}$. $a^{m}-1 \operatorname{div}(a-1)=\sum f+m$. 


\section{Problem 9}

Now we state the propositions:

(14) Let us consider finite 0 -sequences $s_{1}, s_{2}$ of $\mathbb{N}$, and a natural number $n$. Suppose len $s_{1}=n+1$ and for every natural number $i$ such that $i \in \operatorname{dom} s_{1}$ holds $s_{1}(i)=i^{5}$ and len $s_{2}=n+1$ and for every natural number $i$ such that $i \in \operatorname{dom} s_{2}$ holds $s_{2}(i)=i^{3}$. Then $\sum s_{2} \mid 3 \cdot\left(\sum s_{1}\right)$.

Proof: Define $\mathcal{F}$ (natural number) $=\$_{1}{ }^{3}$. Consider $S_{2}$ being a sequence of real numbers such that for every natural number $i, S_{2}(i)=\mathcal{F}(i)$. Define $\mathcal{G}$ (natural number) $=\$_{1}{ }^{5}$.

Consider $S_{1}$ being a sequence of real numbers such that for every natural number $i, S_{1}(i)=\mathcal{G}(i)$.

(15) Let us consider integers $a, b$, and a positive natural number $m$. Then $\sum\left\langle\left(\begin{array}{c}m \\ 0\end{array}\right) a^{0} b^{m}, \ldots,\left(\begin{array}{c}m \\ m\end{array}\right) a^{m} b^{0}\right\rangle=a^{m}+b^{m}+\sum\left(\left\langle\left(\begin{array}{c}m \\ 0\end{array}\right) a^{0} b^{m}, \ldots,\left(\begin{array}{c}m \\ m\end{array}\right) a^{m} b^{0}\right\rangle\lceil m)_{\lfloor 1}\right.$.

(16) Let us consider natural numbers $n, k$. If $n$ is odd, then $n \mid k^{n}+(n-k)^{n}$. The theorem is a consequence of (15).

\section{Problem 10}

Now we state the proposition:

(17) Let us consider a finite sequence $s$ of elements of $\mathbb{N}$, and a natural number $n$. Suppose $n>1$ and len $s=n-1$ and for every natural number $i$ such that $i \in \operatorname{dom} s$ holds $s(i)=i^{n}$. If $n$ is odd, then $n \mid \sum s$.

Proof: $\operatorname{rng}(s+\operatorname{Rev}(s)) \subseteq \mathbb{N}$. If $n$ is odd, then $n \mid \sum s$ by [3, (3)].

\section{REFERENCES}

[1] Grzegorz Bancerek, Czesław Byliński, Adam Grabowski, Artur Korniłowicz, Roman Matuszewski, Adam Naumowicz, Karol Pąk, and Josef Urban. Mizar: State-of-the-art and beyond. In Manfred Kerber, Jacques Carette, Cezary Kaliszyk, Florian Rabe, and Volker Sorge, editors, Intelligent Computer Mathematics, volume 9150 of Lecture Notes in Computer Science, pages 261-279. Springer International Publishing, 2015. ISBN 978-3319-20614-1. doi $10.1007 / 978-3-319-20615-8 \_17$.

[2] Grzegorz Bancerek, Czesław Byliński, Adam Grabowski, Artur Korniłowicz, Roman Matuszewski, Adam Naumowicz, and Karol Pakk. The role of the Mizar Mathematical Library for interactive proof development in Mizar. Journal of Automated Reasoning, 61(1):9-32, 2018. do1 $10.1007 /$ s10817-017-9440-6

[3] Marco Riccardi. The perfect number theorem and Wilson's theorem. Formalized Mathematics, 17(2):123-128, 2009. doi:10.2478/v10037-009-0013-y.

[4] Wacław Sierpiński. 250 Problems in Elementary Number Theory. Elsevier, 1970.

[5] Tetsuya Tsunetou, Grzegorz Bancerek, and Yatsuka Nakamura. Zero-based finite sequences Formalized Mathematics, 9(4):825-829, 2001. 
[6] Li Yan, Xiquan Liang, and Junjie Zhao. Gauss lemma and law of quadratic reciprocity. Formalized Mathematics, 16(1):23-28, 2008. doi 10.2478/v10037-008-0004-4.

Accepted February 26, 2020 